evaluated and clinical events related to safety endpoints were adjudicated by independent medical reviewers.

Results Of 650 patients enrolled across 42 centers (29 in the United States and 13 in Europe), 167 patients with late window presentation were included in this analysis. Baseline characteristics and endpoints are reported in the table and compared to the control arms of DAWN and DEFUSE-3. Late presentation patients in COMPLETE achieved a post-procedure successful revascularization rate of $83.2 \%$ (139/167), 90day good functional outcome rate of 55.4\% (87/157), and 90day all-cause mortality rate of $14.4 \%$ (24/167). Symptomatic ICH occurred in $4.2 \%(7 / 167)$ of patients, procedure-related SAEs in $5.4 \%(9 / 167)$ of patients, and no device-related SAE were observed.

Conclusion For patients with late presentation stroke, aspiration thrombectomy with the Penumbra System in anterior circulations is safe and effective. Rates of good functional outcome and all-cause mortality compare favorably to the DAWN and DEFUSE-3 medical management arms.

Disclosures A. Hassan: 2; C; GE Healthcare, Genentech, Medtronic, Microvention, Penumbra, Stryker, Cerenovus, Viz.

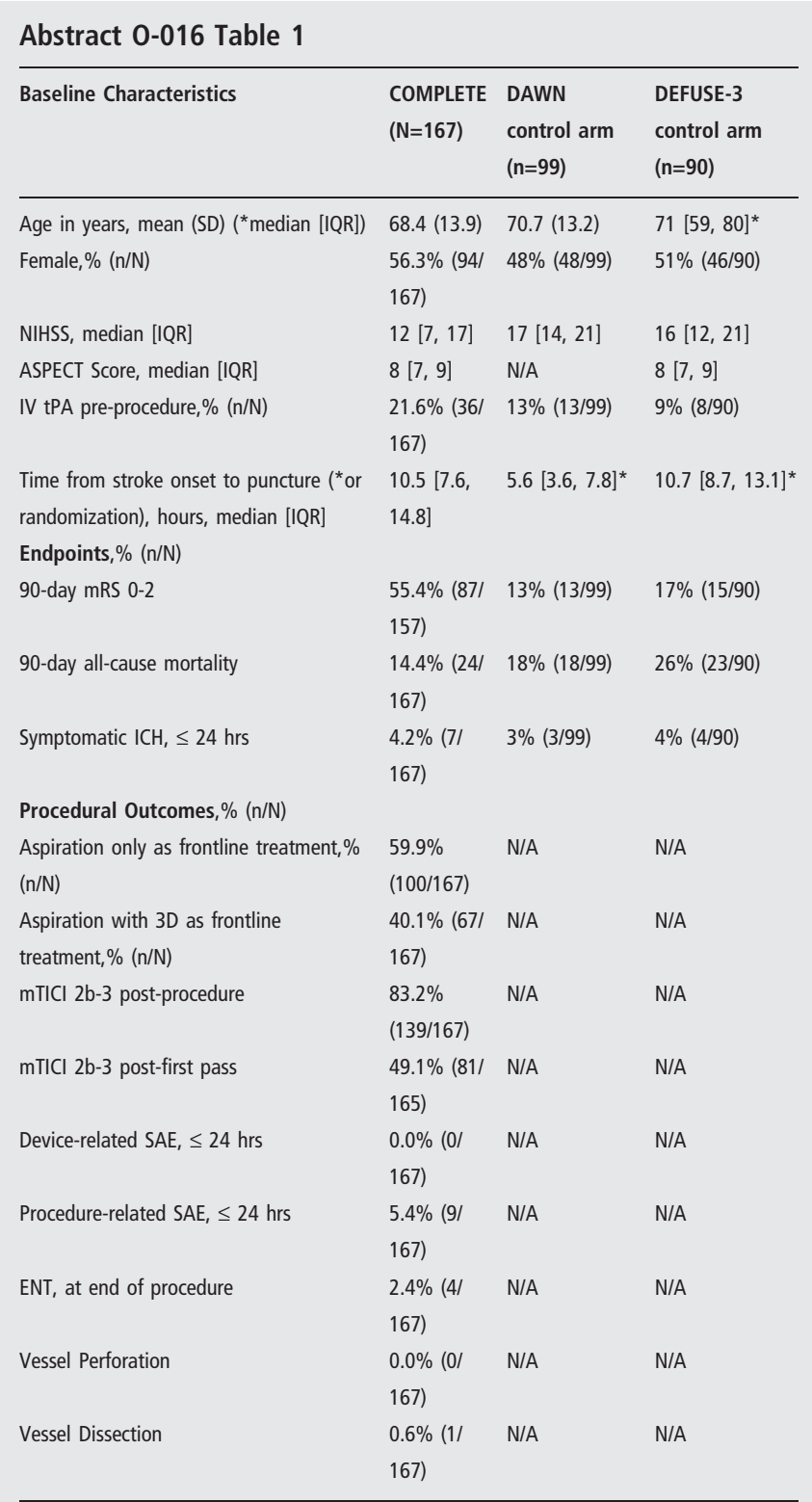

ai, Balt, Scientia. 3; C; GE Healthcare, Genentech, Medtronic, Microvention, Penumbra, Stryker, Cerenovus, Viz.ai, Balt, Scientia. J. Fifi: 1; C; Microvention, Penumbra, Stryker. 2; C; Microvention, Stryker. 4; C; Imperative Care. O. Zaidat: 1; C; Genentech, Medtronic Neurovascular, Stryker. 2; C; Codman, Medtronic Neurovascular, National Institutes of Health StrokeNet, Penumbra, Stryker. 4; C; Galaxy Therapeutics, Inc.

\section{0-017 ADAPT FRONT-LINE THROMBECTOMY- LESSONS LEARNED FROM OVER 1000 STROKES}

${ }^{1} S$ Al Kasab*, ${ }^{2} E$ Almallouhi, ${ }^{1} R$ Chalhoub, ${ }^{3}$ A Alawieh, ${ }^{1} \mathrm{M}$ Sattur, ${ }^{1} \mathrm{G}$ Porto, ${ }^{1} \mathrm{E}$ Bass, ${ }^{1}$ A Spiotta. 'MUSC, Charleston, SC; ${ }^{2}$ Medical University of South Carolina, Charleston, SC; ${ }^{3}$ Emory University, Atlanta, GA

\subsection{6/neurintsurg-2021-SNIS.17}

Introduction Clinical trials have shown that A Direct Aspiration first Pass Technique (ADAPT) is non-inferior to Stentretriever first (SR) technique for mechanical thrombectomy (MT) in stroke patients presenting with emergent large vessel occlusion (LVO). However, longitudinal data regarding the outcomes and evolution of this technique is scarce.

Methods This is a planned analysis of a prospective cohort study that enrolled all patients who underwent ADAPT MT in a comprehensive stroke center between January 2013 and December 2020. We did exploratory analysis of the characteristics and outcomes of patients who had intracranial internal carotid artery (ICA) or M1 segment of middle cerebral artery (MCA) occlusion based on the aspiration catheter bore size (small (041, 054, 058, 060-inch) vs. medium bore (0.064, 0.068 -inch), and medium vs. large bore $(0.071,0.072,0.074$ inch)).

Results During the 8 years study period, a total of 1004 patients were included. Median age was 69, 49\% were females, and $60.6 \%$ were white. Most common LVO was M1 occlusion in 427 (42.5\%) patients followed by M2 occlusion in 217 (21.6\%). Symptomatic hemorrhagic transformation was observed in $47(4.7 \%)$ patients and $366(36.5 \%)$ patients achieved modified Rankin scale (mRS) of 0-2 at 90 days after the stroke. For patients with intracranial ICA or M1 occlusion, medium bore aspiration catheters were more likely to achieve successful recanalization after first aspiration attempt (63.9\% vs. 51.4\%, $\mathrm{P}=0.015$ ), and had a faster groin to reperfusion time (16 vs. 20 min, $\mathrm{P}=0.001$ ) when compared with small bore catheters. However, these differences were not significant when comparing medium to large bore catheters (figures 1 and 2).

Conclusion This study provides a longitudinal overview of the ADAPT MT technique. Medium bore catheters had better performance measures compared to small bore catheters.

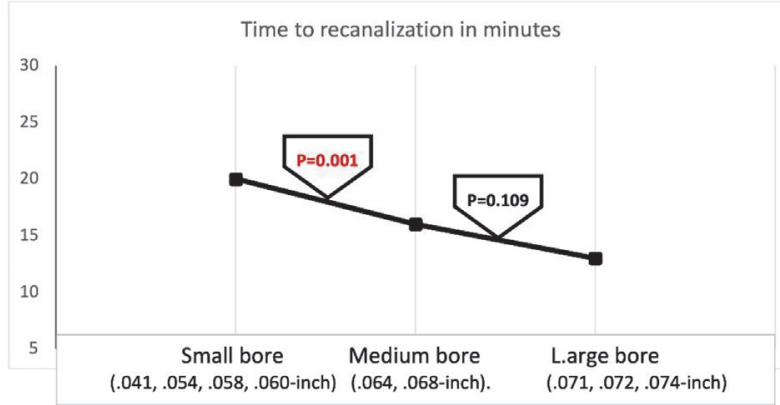

Abstract 0-017 Figure 1 


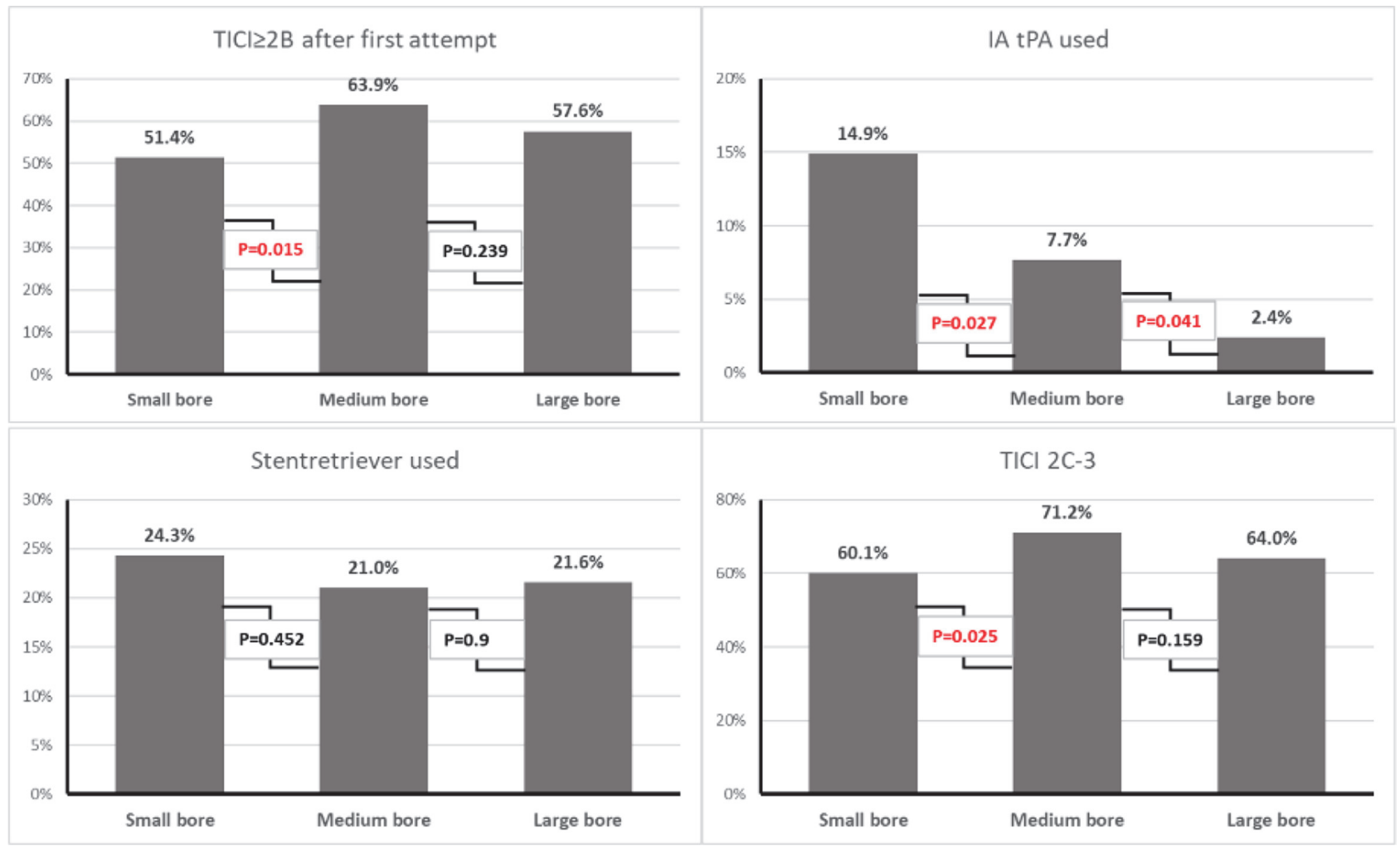

Abstract 0-017 Figure 2

However, large bore catheters did not show significantly better performance results which suggest a plateau effect.

Disclosures S. Al Kasab: None. E. Almallouhi: None. R. Chalhoub: None. A. Alawieh: None. M. Sattur: None. G. Porto: None. E. Bass: None. A. Spiotta: 1; C; Stryker, Penumbra, and Medtronic. 2; C; Stryker, Penumbra, and Terumo.

\section{0-018 THROMBECTOMY OUTCOMES IN PATIENTS WITH LOW ASPECTS TREATED WITHIN 24 HOURS OF SYMPTOMS ONSET: INSIGHTS FROM STAR}

'Z. Hubbard*, 'G. Porto, 'S. Al Kasab, 'E. Almallouhi, ${ }^{2}$ A. Alawieh, ${ }^{3}$ R. De Leacy, ${ }^{4} \mathrm{D}$. Raper, ${ }^{5} \mathrm{~A}$. Rai, ${ }^{6} \mathrm{~T}$. Dumont, ${ }^{7} \mathrm{~S}$. Wolfe, ${ }^{8} \mathrm{P}$. Jabbour, ${ }^{9} \mathrm{C}$. Ogily, ${ }^{10 \mathrm{P}}$. Park, ${ }^{11} \mathrm{M}$. Levitt, ${ }^{12} \mathrm{~A}$. Polifka, ${ }^{13} \mathrm{R}$. Crowley, ${ }^{14} \mathrm{~A}$. Arthur, ${ }^{15} \mathrm{~J}$. Osbun, ${ }^{16} \mathrm{R}$. Crosa, ${ }^{17} \mathrm{I}$. Maier, ${ }^{18} \mathrm{~K}$. Burchiel, ${ }^{2}$ J. Grossberg, ${ }^{19} \mathrm{~S}$. Chowdhry, ${ }^{20} \mathrm{M}$. Mokin, ${ }^{21} \mathrm{C}$. Matouk, ${ }^{22} \mathrm{R}$. Williamson, ${ }^{23} \mathrm{~A}$. Yoo, ${ }^{24} \mathrm{~J}$. Mascitelli, ${ }^{25} \mathrm{P}$. Kan, ${ }^{26} \mathrm{M}$. Psychogios, ${ }^{27} \mathrm{R}$. Starke, ${ }^{1} \mathrm{~A}$. Spiotta. ${ }^{1}$ Medical University of South Carolina, Charleston, SC; ${ }^{2}$ Emory University, Atlanta, GA; ${ }^{3}$ Mount Sinai, New York, NY; ${ }^{4}$ Baylor Medicine Neurosurgery, Houston, TX; ${ }^{5}$ WVU Medicine Neurosurgery, Morgantown, WV; ${ }^{6}$ University of Arizona Neurosurgery, Tuscon, $A Z_{;}{ }^{7}$ Wake Forest Baptist Health Neurosurgery, Lexingon, NC; ${ }^{8}$ Thomas Jefferson University Neurosurgery, Philadelphia, PA; ${ }^{9}$ Beth Israel Hospital-Neurosurgery, Boston, MA; ${ }^{10}$ University of Michigan, Ann Arbor, MI; ${ }^{11}$ UW Neurosurgery, Seattle, WA; ${ }^{12}$ UF Department of Neurosurgery, Gainesville, FL; ${ }^{13}$ RUSH Neurosurgery, Chicago, $\mathrm{LL}_{i}{ }^{14}$ Semmes Murphey Neurosurgeon, Memphis, TN; ${ }^{15}$ Washington University of Saint Louis, St. Louis, MO; ${ }^{16}$ Hospital de Clinicas, Facultad de Medicina, Universidad de la República, Montevideo, Uruguay; ${ }^{17}$ Loma Linda Neurosurgery, Loma Linda, CA; ${ }^{18} \mathrm{OHSU}$ Neurosurgery, Portland, OR; ${ }^{19}$ Northshore, Evanston, $\mathrm{IL}_{i}{ }^{20}$ USF Department of Neurosurgery, Tampa, $\mathrm{FL}_{i}{ }^{21}$ Yale Neurosurgery, New Haven, $C T ;{ }^{22}$ Allegheny Neurosurgery, Pittsburgh, PA; ${ }^{23}$ Texas Stroke Institute, Plano, $T X$; ${ }^{24}$ UT San Antonio, San Antonio, TX; ${ }^{25}$ Medical University of South Carolina, Galveston, $T X_{\text {; }}$ ${ }^{26}$ University Medical Center Göttingen, Göttingen, Germany; ${ }^{27}$ University of Miami Neurosurgery, Miami, FL

10.1136/neurintsurg-2021-SNIS.18
Introduction Outcomes of mechanical thrombectomy (MT) depends largely of achieving reperfusion of a salvageable brain tissue. Patients with poor baseline images were excluded from most clinical trials so the data about their outcomes remain scarce and whether these patients could still benefit from MT remains unknown. In this study, we aim to investigate the safety and efficacy of EVT in patients with large vessel occlusion and large core infarct.

Methods The Stroke Thrombectomy and Aneurysm Registry (STAR) that combined the prospectively maintained databases of 28 thrombectomy-capable stroke centers in the US, Europe, and Asia was interrogated. We included thrombectomy patient presenting with emergent large vessel occlusion within 24 hours and with a large core infarct as defined by Alberta Stroke Program Early CT Score (ASPECTS) < 6. Patients presenting within 6 hours of last known normal (LKN) were considered in the early window and patients presenting after 6 hours were considered in the late window. 90-day outcomes were assessed using modified Rankin scale (mRS)We used a logistic regression model to assess the factors associated with good 90-day outcome in patients in the early and late thrombectomy window.

Results 144 patients were included in this study (table). Median age was 69 and $92(64 \%)$ patients were treated in the early MT window. M1 was the most common site of occlusion (51.4\%) and ADAPT was used in 34.7\%. Median admission NIHSS was 17.5. Successful recanalization (TICI $>2$ b) was achieved in $84.7 \%$ and median procedure time was 54 minutes. sICH hemorrhage was observed in 22 (15.3\%). Regarding 90-day outcome, median mRS was 4, favorable outcome (mRS 0-2) was observed in 41 patients $(28.5 \%)$ and mortality occurred in in 59 (41\%). There was no difference in 90-day functional outcome between patients 